\title{
Musikausbildung im Post-Habsburger Raum (am Beispiel der Musikinstitutionen in Lviv/Lemberg)
}

\author{
Luba Kijanovska, Zoryiana Lastovecka \\ Univerza v Lvovu \\ Universiät Lwiw
}

Vor allem sollte man sich rechtfertigen, warum Lemberger Musikausbildung in der Zwischenkriegsperiode als das „Post-Habsburger“ Modell bezeichnete, weil auf den ersten Blick solche Definition etwa als ein Paradox angenommen sein könnte. Aber man versucht gewählte Definition etwas ausführlicher zu erklären und genanntes Modell als zuverlässige für diese mehrnationale Stadt mit der reichen Kulturtradition darzustellen. Man sollte dabei die ganze Reihe von Bedingungen in Betracht ziehen, um die Situation in damaliger Lemberger Kultur richtig zu verstehen.

Erstens: die österreichische Geschichte, die in Galizien fast 150 Jahre dauerte, war schon im Jahre 1918 vorbei, zu dieser Zeit gehörte das Land zu II Rzeczpospolita, also zum wieder entstandenen polnischen Staat.

Zweitens, österreichische Volksschicht war dementsprechend ganz gering, wenn noch jemand blieb, integrierte sich meistens entweder ins polnische oder ins ukrainische Milieu. Dies hielt auch für die regionale Musikkultur, wie auch für solchen ihren wichtigen Teil als Musikausbildung.

Drittens, man muß berücksichtigen, daß auch soziale und finanzielle Bedingungen dieser Periode gar nicht günstig gewesen sind. Schwere ökonomische Krise zoer Jahre des XX Jh. ließ ihren schmerzhaften Spur in allen Sphären der Kultur und Ausbildung, die Konflikte zwischen Polen und Ukrainer explodierten hier regelmäßig, es widerspiegelte auch gewissermaßen im Kunstleben (obwohl die Auseinandersetzung der Vertreter 
verschiedener Nationalkreisen in den Musikinstitutionen viel weniger und milder als in anderen gesellschaftlichen Sphären war).

Letztendlich wurde Lemberg statt seiner früheren Position der Hauptstadt des Königtums Galizien und Lodomerien allmählich zur Provinz im Vergleich zu Warschau. Mehrere bedeutende Wissenschaftler, Künstler, Professoren sind nach Warschau, wie auch nach anderen größeren Städten Polens, umgezogen. Alle diese Voraussetzungen damaliger Musikausbildung in Lemberg scheinen kaum mit den materiellen Bedingungen der Kultur- und Kunstinstitutionen im Habsburger Imperium vergleichbar.

Alle diese Bedingungen haben wesentlich die Entwicklung der Musikkultur und mehrere wichtige Aktivitäten in diesem Gebiet eingeschränkt. Manche wichtige Institutionen Pleite gehen zu lassen. So, z. B. in Jahren 1931-32 wurde die Musikabteilung des Stadttheaters wegen der schweren finanziellen Lage in Polen geschlossen. Die Musikaufführungen könnten man nur als private Initiative den „Verein der Freunde der Musik und Oper“ (Towarzystwo Miłośników Muzyki i Opery). Dieser Verein wurde von Tadeusz Majerski, Lemberger Komponist und Pädagoge, im Jahr 1931 gegründet. ${ }^{\prime}$ Aber insofern dieser Verein keine Opernaufführung vorbereitet hat, nur die symphonischen Konzerte, bald hörte die Oper ihre mehr als 150-Jährige Existenz in Lemberg auf. Nächste vier Jahre fanden auf der Bühne des Stadttheaters (oder, wie hat es öfter genannt, des Großen Theaters Lemberg) nur gelegentliche Gastaufführungen oder (auch ziemlich seltene) symphonische Konzerte statt. ${ }^{2}$ Unter den Gastaufführungen sind die Auftritte der Operntruppe aus Possen im September 1935 erwähnt. ${ }^{3}$ Von demselben Jahr wirkte die Operabteilung des Großen Theaters nach dem Stagionesystem, d. h. eine oder höchstens 2-3 Oper während paar Monate pro Saison. ${ }^{4}$ Die Opernstagionen begonnen auf der Bühne des Stadttheaters im September 1935.

$1 \quad$ Małgorzata Komorowska, Teatr muzyczny drugiej Rzeczypospolitej (Warszawa: AMFC, 1997), 99.

2 Oxana Palamartschuk, „Львівському театру Опери і Балету 10о“ [Das Lviver Theater für Oper und Ballett feiert 100 Jahre], in: Світ моїх зацікавлень [Die Welt meiner Interessen] (Lviv: Spolom, 2006), 271. Alfred Plohn, „Z teatru Wielkiego”, Chwila, no. 5908 (1935): 11.

4 Leszek Mazepa, „Загальний огляд музичної культури Східної Галичини до $1939^{\prime \prime}$ [Allgemeine Übersicht der Musikkultur der Ostgalizien vor 1939], in: Leszek Mazepa, Сторінки музичного минулого Львова [Blätter musikalischer Vergangenheit Lviv's] (Lviv: Spolom, 2001), 117. 
Obengenannten Tatsachen zeugen davon, daß objektive existentielle und soziale Sachverhalte gar nicht günstig für die Musikinstitutionen in Lemberg und ganz Galizien gewesen sind. Aber trotzdem entwickelte sich die Musikausbildung auf allen Niveaus in der Region sehr rasch, zeigte beachtenswerte Leistungen und könnten mehrmals manche professionelle Musikkollektive, z. B. Operntruppe oder symphonische Orchester der Philharmonie, die wegen der Mangel an Finanzen stabil nicht wirken können, im regulären Kulturbetrieb ersetzen.

Man muß in Zusammenhang mit diesem Thema (und je mehr - mit dieser Formulierung des Themas) noch eine Frage beantworten. Inwiefern unterschied das „Habsburg-Modell“ der Musikausbildung von den anderen künstlerisch hochentwickelten Ländern, etwa von Frankreich, England und besonders von Deutschland? Das neuste Österreichische Musiklexikon bestätigt solche bedeutende -nicht nur für das Land schlechthin, sondern auch für mehrere ehemalige Provinzen des mehrnationalen Habsburger Imperiums - Merkmale der Musikausbildung im Österreich:

Die Entstehung der bürgerlichen Musikkultur führte zu Beginn des 19. Jahrhunderts zur Gründung von Vereinigungen, welche sich neben der Aufführung aufwendiger Werke auch die Hebung des musikalischen Niveaus zum Ziel setzten (Musikverein, Kirchenmusikschulen), wobei zunächst die Laienausbildung (Dilettant), nicht die Ausbildung professioneller Musiker im Vordergrund stand. Aus diesem Antrieb kam es innerhalb weniger Jahre zur Gründung von Sing- bzw. Musikschulen (Graz 1816, Wien 1817, Innsbruck 1818, Linz 1823, Klagenfurt 1828). In der zweiten Hälfte des 19. Jahrhunderts entwickelten sich diese Musikschulen, allen voran das Konservatorium der Gesellschaft der Musikfreunde in Wien, zu professionellen Ausbildungsinstitutionen, welche auch die künstlerischen Bedürfnisse des bürgerlichen Musiklebens zu erfüllen begannen. ${ }^{6}$

Man könnte vermuten, genau dieselben Prozesse im Gebiet der Musikausbildung fanden in mehreren europäischen Staaten bzw. Imperien statt. Welche Spezifik bezeichnete gerade das österreichische System? Ich erlaube mir in diesem Zusammenhang eigene Hypothese vorschlagen.

Erste und etwa wichtigste Besonderheit besteht darin, daß das Österreich mehr als irgendeiner andere Staat des späten 18. - 19. Jahrhunderts

6 Gabriele Eder, „Musikausbildung,“ in: Oesterreichisches Musiklexikon online, https://www.musiklexikon.ac.at/ml/musik_M/Musikausbildung.xml. 
mehrnational, dementsprechend mehrkonfessionell und „mehrtraditionell“ (auch im Sinne der Musikkultur) war. Es führte unentbehrlich dazu, daß diese verschiedenen Traditionen miteinander zusammenwirkten, einander beeinflussten, auch im Gebiet der Musikausbildung (z. B. im Repertoire, im Lehrkreis, insofern in derselben Institution die Vertreter verschiedener Nationalschichten nebeneinander wirkten). Aber es bedeutete gar nicht, daß eigene nationale Musiktraditionen in der Ausbildungspraxis vernachlässigt sind und den allgemeinen künstlerischen und weltanschaulichen Werten der Metropolie absolut untertan sind. Genau umgekehrt, eigene geistliche Ideale sind noch sorgfältiger gepflegt, das Kulturgut anderer Völker und vor allem die Kultur der Hauptstadt Wien diente für die wechselwirkende Bereicherung und den Fortschritt jeder von nationalen Traditionen. Am Lemberger Konservatorium des Galizischen Musikvereins (GMV), welches 1853 offiziell gegründet wurde, obwohl viel älter als Lehranstalt funktionierte (etwa 1844 als die Musikschule mit mehreren Fächern), sah dieser Prozeß so aus, daß ein Korpus von Pädagogen in der zweiten Hälfte des XIX Jh. die Vertreter verschiedener Nationalitäten bildeten: der erste Direktor - der österreichische Pianist und Komponist französischer Herkunft Johannes Ruckgaber, sein Nachfolger, Armenier, Pianist und Komponist Karol Mikuli, der bei Chopin studierte, dementsprechend als polnischer Musiker sich identifizierte; zahlreiche Polen, u. a. der Sänger Waleri Wysocki und der nächste Direktor Mieczysław Sołtys, der italienische Sänger Luigi della Casa, der slowenische Dirigent und Komponist Fran Gerbič, der tschechische Organist und Komponist Rudolf Schwarz, wirkte der Ukrainer Anatol Wachnianyn mit u. m. a. Nicht minder bunt war auch die Liste von Studenten. Darüber hinaus sollte man besonders betonen, daß das Konservatorium am GMV wie auch andere Musiklehranstalten in der Stadt waren nie antisemitisch, z. B. bei Mikuli studierten solche Pianisten und Pianistinnen wie Moriz Rosenthal, Kornelia Parnas-Löwenherz, Klara Gescheles, bei Wysocki - Joseph Mann, Roza Cudek u. a., hier arbeitete der Theoretiker Seweryn Barbag.

Diese langjährige national-kulturelle Verschmelzung bedingte manche feste Traditionen der Musikausbildung im Land, die für nächste Generationen auch gültig werden, wie die Toleranz der Pädagoge und Studenten abgesehen von nationaler Herkunft (man sagt oft bis heute, daß am Lemberger Konservatorium nur eine Nationalität man berücksichtigte das Talent); das Interesse für breiten Kreis von verschiedenen nationalen Leistungen, die nicht nur in den großen Kulturzentren entstanden, son- 
dern auch in „peripheren“ Ländern; die Offenheit für die musik-pädagogischen Innovationen.

Noch eine spezifisch österreichische historisch-politische Entscheidung brachte weitgehende Ergebnisse, vor allen Dingen in der Kultur und Ausbildung mehrerer Völker, die im Habsburger Imperium lebten. Es geht um die national-kulturelle Autonomie (1867), nach der eine echte Blütezeit für die verschiedene nationale Chor- und Musikvereine, für andere Musikinstitutionen, darunter Musikschulen und Konservatorien, herkam. Dies erklärt, warum polnische und ukrainische Bevölkerung Galiziens viel intensiver ihre eigene Musikkultur (wie auch andere Aktivitäten in der humanitären Sphäre) entwickelten, als die Polen und Ukrainer im Rußland oder Polen in Preußen.

Alles, was in der auf gegenwärtigen Stufe befindlichen Verwaltungstätigkeit des Staates unmittelbar die Pflege der Nationalität berührt, muß der nationalen Selbstverwaltung überliefert, beziehungsweise der "internationalen" Majoritätsgewalt entzogen werden. ${ }^{7}$

Hier muß man speziell unterstreichen, daß Galizien, vor allem polnische Bevölkerung des Landes, aber auch gewissermaßen die Ukrainer - Ruthene, besondere Rechte in ihren nationalen Kultur- und Ausbildungsbedürfnissen erreicht haben. Bis heute wurde relativ größere national-kulturelle Freiheit des Landes zum Symbol der kulturellen Autonomie im ganzen Imperium: „Der Name Galizien symbolisiert heute vor allem die kulturelle Autonomie, die diese Region während der Teilungszeit besa $\beta^{\text {“. }}{ }^{8}$ Selbstverständlich alle nationale Ausbildungsinstitutionen, darunter Musiklehranstalten strebten zu der breitesten Ausnutzung solcher Möglichkeiten. Dies führte bald zur Gründung mehrerer neuen Institutionen solcher Art, die auch nach dem Niedergang des Habsburger Imperiums ihre Tätigkeit fortsetzten.

Anfangs des XX Jh. wurde in Lemberg nach den vieljährigen Bemühungen noch eine, schon rein ukrainische, Musiklehranstalt gegründet:

7 Alfredvon Offermann, Die Verfassungalsdie Quelle des Nationalitätenhadersin Österreich. Studie eines Patrioten (Wien, Leipzig: 1897), 74.

8 „Galizien“, Länder \& Regionen, Deutsches Kulturforum Östliches Europa, http:// www.kulturforum.info/de/startseite-de/1019467-laender-regionen/100oo82-polen/1019489-galizien. 
das Hohe Musikinstitut (Вищий Музичний Інститут). Sein erstes Studienjahr begann 10 Oktober 1903. ${ }^{9}$

In diesem Kontext muß man auch die Begründung der Musikschule von Anna Niementowska im Jahr 1902 erwähnen, die später im Jahr 1932 zum Szymanowski Konservatorium umgestaltet wurde.

1912 begann seine fruchtbare etwa 30-jährige Tätigkeit das Lehrstuhl für Musikwissenschaft oder, wie es öfter genannt wurde, Musikwissenschaftliche Lehranstalt (Zakład Muzykołogiczny) an der Lemberger Universität unter der Leitung von bedeutendem polnischem Musikforscher Professor Adolf Chybiński.

Also vor dem Ersten Weltkrieg wirkten in der Stadt vier Musikausbildungsinstitutionen höherer Stufe, dazu 63 Musikschulen verschiedener Spezialisierung, von mehrdimensionalen Lehranstalten mit der imponierenden Zahl von Zöglingen und mit der staatlichen Subvention - bis zu kleinen privaten Schulen, wo nur ein Musikfach geübt wurde. ${ }^{10}$

Die dritte spezifische Eigenschaft des österreichischen Modells der Musikausbildung besteht darin, daß alle derartige Institutionen meistens sehr eng mit den Anfragen und Bedürfnissen der Gesellschaft verbunden waren, da meistens mit den verschiedenen musikalischen Amateur-Vereinen, also sozial gerichteten Vereinigungen verbunden sind. Dementsprechend entfalteten die Aktivitäten der Musikschulen und Konservatorien strikt an einem konkreten Ort und zu einem konkreten Zeitpunkt, in voller Übereinstimmung mit den sozialen Anforderungen - davon wurde doch ihre finanzielle Lage abgehängt. Denn der Anteil der Pädagogen und Studenten im offenen Musikleben sollten volens nolens sehr aktiv sein, auf die Anfrage der Gesellschaft mußte man immer sehr aufpassen, dadurch die Beziehung zwischen den Musiker-Liebhabern - professionellen Musikern und der übrigen Gesellschaft war immer sehr intensiv und wechselwirkend.

Wichtige Konzertveranstalter (auch für Abendunterhaltungen) wurden in der Folge die Musikvereine. Stand zunächst noch das gemeinsame Musizieren der Mitglieder im Vordergrund und bildeten

9 Dieses Datum findet man im Bericht von der Entwicklung der Hohen Musikinstitut [Звіт з розвою Висшого Музичного Інститута. Lemberg Львів, 1904, 1]. Die Lemberger Zeitung „Die Sache” („Діло”) von 3(16). Х. 1903 p. gab das Datum 1 Oktober. Zit. nach: Leszek Mazepa, „Allgemeine Übersicht“, 124. 
oft solche Liebhaberkonzerte den Auslöser zur Vereinsgründung, so verschob sich der Schwerpunkt ihrer Tätigkeit immer mehr in Richtung musikpädagogischer (Musikvereinsschulen, Konservatorium) und organisatorischer (Veranstaltung von Konzerten) Aufgaben, die Entwicklung ging also in Richtung Professionalisierung des Musiklebens. ${ }^{\text {II }}$

Denn könnte man bestätigen, daß das gut organisierte zu Habsburger Periode österreichische Ausbildungssystem zusammen mit den günstigen regionalen Umständen ein fester Grund für weitere fruchtbare Tätigkeit der Musikausbildung in Lemberg und ganz Galizien legte. Damit vermutlich könnte man solches Phänomen erklären, wie trotz der Krise so erfolgreich wirkten in zoer und besonders in $30 e r$ Jahren nicht nur alle vier obengenannten führenden Musikhochschulen, sondern auch mehrere andere; auf welche Weise können ihre Kollektiven und Solisten fast ganzes Musikleben der Stadt auf ihren Schultern tragen; welch eine Zauberkraft schützte die Pädagoge und Studenten aller genannten Lehranstalten vor den nationalen Konflikten, die in anderen gesellschaftlichen Schichten so hart explodierten. Meiner Meinung nach, die frühere, zu österreichischen Zeiten gestaltete fruchtbare Zusammenarbeit in den verschiedenen Musikinstitutionen, vor allem in den Hochschulen, hatte so tiefe Wurzeln und brachte so gute Ergebnisse mit, daß auch später scheint man nicht vernünftig dieses Modell abzulehnen.

Am Ende führe ich manche überzeugende Beispiele, die die vorliegenden theoretischen Nachweisungen illustrieren sollten.

Man beginnt von dem Konservatorium des Polnischen Musikvereins, als der größten solcher Institution im Land. Die Zahl von Studenten des Konservatoriums änderte sich von über 300 in Studienjahren 1933/341937/38 bis 828 in 1924/25 und 1106 in 1927/28 Studienjahr. Bis 1929 führte das Polnische Musikverein und sein Konservatorium Mieczysław Sołtys. Er setzte fort und entwickelte erfolgreich die Traditionen seines Lehrers und Vorgängers Karol Mikuli, gleichzeitig strukturierte das Lernprozeß, führte neue Disziplinen und unterstützte vor allem die Aufführungen polnischer Musik, darin auch der neuen Nationalmusik, gründete - gleich nach dem Ende des Kriegs! - eine Opernschule. Sie wirkte von 1922 beim Konservatorium PMV unter der Leitung des bekannten Sängers, des Solisten Lemberger Oper, Professoren Czesław Zaremba. Als Dirigent genann-

11 Barbara Boisits, „Bürgerliche Musikkultur,“in: Oesterreichisches Musiklexikon online, https://www.musiklexikon.ac.at/ml/musik_B/Buergerliche_Musikkultur.xml. 
ter Opernschule war Adam Sołtys tätig. Bloß mit den Kräften von Pädagogen und Studenten des Konservatoriums sind solche anspruchsvolle Oper wie „Don Giovanni” (1923), „Cosi fan tutte” (1927), „Die Entführung aus dem Serail” (1933) von W. A. Mozart, „Aida” (1928) von G. Verdi, „Fidelio” (1929) von L. van Beethoven, „Il Barbiere die Seviglia” (1932-1933) von G. Rossini, „La Boheme” (1933) von G. Puccini etc.

Der Sohn von Mieczyslaw Sołtys Adam kam zur Leitung der ganzen Institution im Jahr 1929 und bekleidete diese Stelle bis 1939, also in den schwersten Krisenjahren.

Wie erwähnen in seinem Buch Leschek und Teresa Mazepa,

Nach dem Tod von Mieczystaw Soltys im Jahr 1929 übernahm die Leitung des Konservatoriums (des Polnischen Musikvereins - L.K.) sein Sohn Adam Soltys. Dieser hochgeschätzte und professionelle Musiker setzte wichtigste Traditionen seines Vaters im Lernprozeß fort. Obwohl in jener sehr schwierigen Periode scharfer ökonomischer Krise die Zahl von Studenten und Pädagogen relativ weniger war, auch manche Lehrprogramme sollte man kürzen, trotzdem das Konservatorium entwickelte sich weiter und blieb nach wie vor die Stütze der Musikausbildung in Galizien. ${ }^{\text {I2 }}$

Was scheint besonders bemerkenswert, die Aufführungen des Opernstudiums fanden regulär auch in zoer Jahre statt, wann, wie obenerwähnt, eine ständige Operntruppe ging pleite. So berichtete die Presse

Dank der Tätigkeit von Adam Soltys hörte Lemberg,Die lustigen Weiber von Windsor'von Otto Nicolai. Die Opernschule des Polnischen Musikvereins und das Orchester der Philharmonie unter der Leitung von Adam Soltys schenkten den, durstigen' nach der Oper Zuhörern (in einem übervollen Saal!) die Aufführung, die von das Niveau dieser Hochschule am besten zeugte. ${ }^{13}$

Nicht nur das Konservatorium des Polnischen Musikvereins zeigte in der Zwischenkriegsperiode solche bedeutende Leistungen. Alle anderen obengenannten Hochschulen rivalisieren mit ihm ziemlich erfolgreich. So z. B. das Musikinstitut unter der Leitung von Anna Nementovska gründete

12 Leszek Mazepa und Teresa Mazepa, Шлях до музичної академії у Львові [Der Weg zur Musikakademie in Lviv], Bd. 1 (Lviv, Spolom, 2003), 204.

13 Maria-Ewa Sołtys, Tilko we Lwowie. Dzieje życia I działalności Mieczysława i Adama Sołtysów (Wrocław-Warszawa-Kraków: Zakład Narodowy im. Ossolinśkich, 2008), 127. 
noch vor dem Ersten Weltkrieg im Jahr 1910 die Filiale in kleineren Städten Galiziens, wie auch das Seminar für Musiklehrer. In 2oer Jahren sind noch die Opernschule und die dramatische Schule an diesem Institut geschaffen. Die Leitidee des Musikinstituts war: „Innovation und Professionalismus”. Frau Direktorin Niementowska noch im Jahr 1923 reiste nach USA, Frankreich, England und Schweiz, verblieb dort eineinhalb Jahre, um verschiedene neue musikpädagogische Systeme zu erforschen und in heimischer Lehranstalt ihre werteste Elemente zu übernehmen.

Ganz gesetzmäßig wurde das Musikinstitut unter der Leitung von Anna Niementowska im Jahr 1931 ins Lemberger Musikkonservatorium „Karol Szymanowski” umgestaltet. Diese Ehre hat sie mit vollem Recht verdient, weil hier sehr erfolgreich das symphonische Orchester und der Chor der älteren Schüler, wie auch das Kinderorchester und der Kinderchor wirkten. Die Kinderkollektive bildeten die Zöglinge der sog. „Fröbel-Musikschule", die sich als eine einzelne Abteilung bei der Schule von Niementowska noch von 1910 entpuppte. Welch ein Niveau erreichte das symphonische Orchester, zeugt z. B. das Programm eines Konzertes von Jahr 1932, unter der Leitung des bekannten polnischen Komponisten und Dirigenten Grzegorz Fitelberg: symphonische Dichtung „Till Eulenspiegel” von Richard Strauss, die symphonische Episode „Die Eisengießerei“ von Aleksander Mosolow, der zweite Teil des Balletts „Harnasi” von Karol Szymanowski, Sinfonia brevis und zwei Liederzyklen von Tadeusz Jarecki, wie auch das Klavierkonzert f-Moll von Fryderyk Chopin ${ }^{\mathrm{I}}$.

Sehr erfolgreich entwickelte sich das Hohe Musikinstitut (von 1912 das Hohe Musikinstitut „Mykola Lyssenko”), welches aufgrund des ukrainischen Chorvereins „Bojan ${ }^{\text {“Is }}$ und anderer ukrainischen Musikvereine im Jahr 1903 gegründet wurde. In diesem Zusammenhang scheint sehr interessant, daß der Begründer und erster Direktor Anatol Wachnianyn aus der ukrainisch-tschechisch-österreichische Familie stammte, in Wien studierte, mit dem polnischen Musikverein „Laute“ (Lutnia) mitwirkte, parallel mehrere ukrainische nationale Kultur- und Ausbildungsinstitutionen und Vereinen ins Leben gerufen hat. Als erste Pädagoge des Instituts sind außer den Ukrainern und Ukrainerinnen - Olena Jasenytska, Maria Krynytska (Klavier) u. a., ein geborener Tscheche, der der polnischen Kultur sich widmete, Jan Gall (Harmonie und Kontrapunkt), der österreichische Violinist Otto Teitsch eingeladen. Obwohl die erste Periode seiner Tätigkeit ziem-

\footnotetext{
14 Mazepa und Mazepa, Der Weg zur Musikakademie, 206.

15 Bojan - ein legendärer altruthenischer Sänger.
} 
lich bescheiden war, bald erreichte das Hohe Musikinstitut - wieder trotz den höchstens ungünstigen Umständen, trotz dem Krieg und nächsten politischen Schwierigkeiten - sehr angesehene Position nicht nur in Lemberg und im Land, sondern auch in ganz Polen. Seine Leitung übernommen die Absolventen der führenden europäischen Konservatorien - Stanislav Ludkewytsch, Direktor des Instituts in Jahren 1910-1915, später sein Inspektor, studierte in Wien bei Aleksander Zemlinski, Hermann Graedener und Guido Adler, Filaret Kolessa, einer der ersten Professoren des Instituts - auch in Wien bei Anton Bruckner und Vatroslav Jagić, später in Berlin bei Erich von Hornbostel; Vassyl Barvinskyj, Direktor des Instituts in Jahren 1915-1939 - am Prager Konservatorium bei Vitězslav Novák und Jakob Golfeld.

Unter solcher professionellen Leitung gestaltete sich das Institut in 2oer - 3oer Jahre als die Lehranstalt nach den besten europäischen Konservatorien-Mustern, vor allem in Wien und Prag. Alle drei Ebene der Studien - die Grundstufe (erste 4 Jahre), Mittelstufe (weitere 4 Jahre) und Meisterstufe (letzte 2 Jahre) - enthielten alle Instrumente und gaben gründliche theoretische Vorbereitung. Institut, dessen Hauptgebäude in Lemberg befand, öffnete während der 2oer - 3oer Jahre noch 13 Filialen fast in allen Städten und Städtchen des Landes, in 3oer Jahren - noch die Dirigentenkurse und musikpädagogische Kurse. Die Zahl der Studierenden beträgt zu 1939 über 6oo Personen, die Zahl der Pädagoge - über 6o.

Also, schon diese kurze Übersicht der bedeutenden Leistungen der Lemberger Hochschulen für Musik in den schwersten Jahren der ökonomischer Krise zeugt davon, daß das früher tief eingewurzelte erfolgreiche Ausbildungssystem in der Musiksphäre wurde auch später, unter den anderen politischen und sozialen Bedingungen, fruchtbar realisiert.

\section{Bibliographie}

Boisits, Barbara. „Bürgerliche Musikkultur“. In: Oesterreichisches Musiklexikon online. https://www.musiklexikon.ac.at/ml/musik_B/Buergerliche_Musikkultur.xml.

Deutsches Kulturforum Östliches Europa. „Galizien“. Länder \& Regionen. http:// www.kulturforum.info/de/startseite-de/1019467-laender-regionen/1000o82polen/1019489-galizien.

Eder, Gabriele. „Musikausbildung“. In: Oesterreichisches Musiklexikon online. https://www.musiklexikon.ac.at/ml/musik_M/Musikausbildung.xml. 
Komorowska, Małgorzata. Teatr muzyczny drugiej Rzeczypospolitej. Warszawa: AMFC, 1997.

Mazepa, Leszek und Teresa Mazepa. Шлях до музичної академії у Львові [Der Weg zur Musikakademie in Lviv], Bd. 1. Lviv: Spolom, 2003.

Mazepa, Leszek. „Загальний огляд музичної культури Східної Галичини до 1939“ [Allgemeine Übersicht der Musikkultur der Ostgalizien vor 1939]. In: Mazepa, Leschek. Сторінки музичного минулого Львова [Blätter musikalischer Vergangenheit Lviv's]. Lviv: Spolom, 2001.

Offermann, Alfred von. Die Verfassung als die Quelle des Nationalitätenhaders in Österreich. Studie eines Patrioten. Wien, Leipzig: 1897.

Palamartschuk, Oхаna. „Львівському театру Опери і Балету 10о“ [Das Lviver Theater für Oper und Ballett feiert 100 Jahre]. In: Світ моїх зацікавлень [Die Welt meiner Interessen]. Lviv: Spolom, 2006.

Plohn, Alfred. „Z teatru Wielkiego”. Chwila, Nr. 5908 (1935): 11.

Sołtys, Maria-Ewa. Tilko we Lwowie. Dzieje życia i działalności Mieczysława i Adama Sołtysów. Wrocław-Warszawa-Kraków: Zakład Narodowy im. Ossolinśkich, 2008. 